\title{
Loss of autophagy-related protein Beclin 1 may define poor prognosis in ovarian clear cell carcinomas
}

\author{
HIROSHI KATAGIRI ${ }^{1}$, KENTARO NAKAYAMA ${ }^{1}$, SULTANA RAZIA $^{1}$, KOHEI NAKAMURA ${ }^{1}$, EMI SATO ${ }^{1}$, \\ TOMOKA ISHIBASHI ${ }^{1}$, MASAKO ISHIKAWA ${ }^{1}$, KOUJI IIDA ${ }^{1}$, NORIYOSHI ISHIKAWA ${ }^{4}$, \\ YOSHIRO OTSUKI ${ }^{3}$, SATORU NAKAYAMA ${ }^{2}$ and SATORU KYO ${ }^{1}$ \\ ${ }^{1}$ Department of Obstetrics and Gynecology, Shimane University School of Medicine, Izumo 693-8501; \\ ${ }^{2}$ Department of Obstetrics and Gynecology, Seirei Hamamatsu General Hospital, Hamamatsu 430-8558; \\ ${ }^{3}$ Department of Pathology, Seirei Hamamatsu General Hospital, Hamamatsu 430-8558; ${ }^{4}$ Department \\ of Organ Pathology, Shimane University School of Medicine, Izumo 693-8501, Japan
}

Received June 23, 2015; Accepted August 3, 2015

DOI: $10.3892 /$ ijo.2015.3191

\begin{abstract}
The aim of the present study was to clarify the role of autophagy in cisplatin (CDDP) sensitivity in OCCCs and the role of Beclin 1 in OCCC progression. Autophagy was measured using: i) western blot analysis of LC3 and p62 and ii) microscopic observation of GFP-LC3 puncta. Autophagy was suppressed using chloroquine and Beclin 1 siRNA. Surgical specimens were examined for Beclin 1 protein expression by immunohistochemistry. The correlations between the loss of Beclin 1 expression and clinicopathological characteristics, prognosis and chemosensitivity were investigated. Inhibition of autophagy by chloroquine or Beclin 1 siRNA did not enhance the sensitivity of the ES2 and TOV-21G OCCC cell lines to CDDP. Loss of Beclin 1 expression was observed in $38.3 \%(23 / 60)$ of the analyzed tumors. There was no significant correlation between loss of Beclin 1 expression and FIGO stage, CA125 levels, patient age, status of endometriosis, Ki-67 labeling index, chemotherapy regimen or status of residual tumor. However, negative expression of Beclin 1 was associated with a shorter progression-free survival in comparison to positive Beclin 1 expression in OCCC who received cytoreductive surgery, followed by a standard platinum-based chemotherapy regimen ( $\mathrm{P}=0.027$, log-rank test). Beclin 1-negative tumors were no more resistant to primary adjuvant chemotherapy than were Beclin 1-positive tumors (50.0 vs. 66.7\%, $\mathrm{P}=0.937$ ). Beclin 1 knockdown using siRNA increased cell growth but not cell migration and invasion in ES2 and TOV-21G OCCC cell lines. Autophagy defects caused by loss of Beclin 1 are not
\end{abstract}

Correspondence to: Dr Kentaro Nakayama, Department of Obstetrics and Gynecology, Shimane University School of Medicine, Enyacho 89-1, Izumo 693-8501, Japan

E-mail:kn88@med.shimane-u.ac.jp

Abbreviations: CDDP, cisplatin; OCCCs, ovarian clear cell carcinomas

Key words: ovarian clear cell carcinoma, Beclin 1, autophagy related to chemoresistance and metastasis, but may be associated with malignant phenotype and poor prognosis of OCCC.

\section{Introduction}

Ovarian carcinoma is the most lethal gynecological malignancy in American and Japanese women (1). Despite aggressive treatment, most patients eventually experience a recurrence of a chemoresistant form of the disease. Ovarian carcinoma is classified into four molecularly distinct histological types: serous, mucinous, endometrioid, and clear cell (2). Ovarian clear cell carcinoma (OCCC), which constitutes approximately $25 \%$ of ovarian carcinoma in Japan, is highly resistant to conventional platinum based chemotherapy (3). As such, OCCC carries a poor prognosis, despite early-stage diagnosis in $60 \%$ of cases. The evasion of apoptosis is a hallmark of cancer cells (4), and the failure of anticancer treatments to induce apoptosis leads to chemotherapeutic failure and tumor progression. However, the role of autophagy, an alternative caspase-independent cell death program $(5,6)$, and its underlying molecular mechanism in OCCC chemoresistance and tumor progression remain unclear. Autophagy is a process of degradation and recycling of cytoplasmic components for energy utilization. Autophagy also occurs in response to certain forms of therapeutic stress, including cytotoxic chemotherapy $(7,8)$. Approximately 30 yeast genes and 16 human homologues have been identified as autophagy-related genes. Among these genes, Beclin 1 plays key roles in mammalian autophagy $(9,10)$.

To the best of our knowledge, the role of autophagy in chemoresistance and the clinocopahological siginificance of Beclin 1, a key molecule for autophagy induction in OCCCs, have not been examined. The goal of the present study was to clarify the role of autophagy in cisplatin sensitivity in OCCCs and the role of Beclin 1 in OCCC progression.

\section{Materials and methods}

Tissue samples. Formalin-fixed, paraffin-embedded tissue samples of 60 ovarian clear cell carcinomas were used in the 
present study. Samples were obtained from the Department of Obstetrics and Gynecology at the Shimane University Hospital and the Department of Obstetrics and Gynecology at Seirei Hamamatsu General Hospital. Diagnosis was based on conventional morphological examination of sections stained with hematoxylin and eosin (H\&E), and tumors were classified according to the WHO guidelines. Tumor staging was performed using the International Federation of Gynecology and Obstetrics (FIGO) classification system. All patients were primarily treated with cytoreductive surgery and adjuvant platinum and taxane or CPT-11 chemotherapy (CBDCA AUC 5 with paclitaxel $175 \mathrm{mg} / \mathrm{m}^{2}$ or docetaxel $70 \mathrm{mg} / \mathrm{m}^{2}$ or CDDP $60 \mathrm{mg} / \mathrm{m}^{2}$ with CPT-11 $180 \mathrm{mg} / \mathrm{m}^{2}$ ). All patients received 6-12 courses of this combination regimen. Acquisition of tissue specimens and clinical information was approved by the institutional review board of Shimane University \& Serrei Hamamatsu General Hospital. The paraffin tissue blocks were organized into tissue microarrays, each made by removing 3-mm diameter cores of tumor from the block. Selection of the area to core was made by a gynecologic oncologist (K.N) and pathology technician (K.I) and was based on review of the H\&E slides.

Immunohistochemistry. For immunohistochemistry, paraffin sections were deparaffinized and incubated with a primary mouse Beclin 1 antibody (Cell Signaling Technology, Danvers, MA, USA) at a dilution of $1: 100$ in a $4^{\circ} \mathrm{C}$ moist chamber overnight. Two independent observers scored Beclin 1 immunoreactivity using a categorical scoring system from 0 (not detectable) to 3 (intense) with the mean score recorded from triplicate samples. Immunostaining and evaluation of ARID1A (a dilution 1:100; Santa Cruz Biotechnology, Santa Cruz, CA, USA) was previously performed (11).

Mutation analysis. Of the 60 OCCCs, 37 or 56 samples were available for Kras or PIK3CA DNA sequencing respectively, and had been previously evaluated (12). Exons 9 and 20 of the PIK3CA gene and exon 1 of the KRAS gene (including codons 12 and 13) were amplified by polymerase chain reaction (PCR) using primer sets previously described (12). Polymerase chain reaction products were purified using the Qiagen PCR purification kit (Qiagen, Valencia, CA, USA) and used for direct sequencing.

Gene amplification analysis. ZNF217 gene amplification analysis was performed and evaluated as previously described (13). Briefly, BAC clones (RP5-823G15 and RP4-724E16) containing the genomic sequences of the 20q13.2 amplicon for ZNF217 locus were purchased from BACPAC Resource Center (Children's Hospital, Oakland, CA, USA) and Invitrogen (Carlsbad, CA, USA). BAC clones corresponding to the Ch20P centromere (RP5-1025A1 and RP4-738P15) were used to generate reference probes. The method used for fluorescence in situ hybridization (FISH) was described by Nakayama et al (14).

Cell culture and cell lines. ES2 (clear cell carcinoma) and TOV-21G (clear cell carcinoma) human ovarian cancer cell lines were obtained from the American Type Culture Collection (ATCC; Rockville, MD, USA).
Western blot analysis. Western blot analysis was performed on ovarian cancer cell lines ES2 and TOV-21G. Cell lysates were prepared by dissolving cell pellets in Laemmli sample buffer (Bio-Rad Laboratories, Hercules, CA, USA) supplemented with $5 \%$ beta-mercaptoethanol (Sigma, St. Louis, MO, USA). Similar amounts of total protein from each lysate were loaded and separated on $10 \%$ Tris-glycine SDS-polyacrylamide gels (Novex, San Diego, CA, USA) and electroblotted to Millipore Immobilon-P polyvinylidene difluoride membranes. The membranes were probed with Beclin 1 antibody (1:100; Cell Signaling Technology), LC-III (1:1,000; Santa Cruz Biotechnology), or p62 (1:100; Enzo Life Sciences, Inc., Plymouth Meeting, PA, USA) followed by peroxidase conjugated anti-mouse or anti-rabbit immunoglobulin $(1: 20,000)$. The same membrane was probed with a GAPDH antibody $(1: 10,000)$ (Cell Signaling Technology) for loading controls. Western blots were developed using a chemiluminescence kit (Pierce, Rockford, IL, USA).

Silencing RNA knockdown of Beclin 1 gene expression. Beclin 1 and control siRNA (luciferase siRNA) were purchased from Cell Signaling Technology. Cells were seeded into 96-well plates and transfected with siRNAs using Oligofectamine (Invitrogen). Following transfection, cells were collected at $48 \mathrm{~h}$ for western blotting of Beclin 1 protein.

Cell proliferation assay. CDDP was purchased from Enzo Life Sciences. Chloroquine disphosphate was purchased from Sigma. Cytotoxicity of CDDP was measured using a 3-(4,5-dimethylthiazol-2-yl)-2,5-diphenyltetrazolium bromide (MTT) colorimetric assay (Sigma). Cells were seeded into 96-well plates at a density of 3,000 cells/well. Cell number was determined indirectly with an MTT assay (15). Data were expressed as the mean \pm 1 SD of triplicate determinations. An MTT cell growth assay was performed $96 \mathrm{~h}$ after treating the cells with Beclin 1 siRNA or control siRNA. The data were expressed as a percentage of the DMSO control. The mean and standard deviation were obtained from three experiments.

Autophagy assays. Autophagy was measured using: i) western blot analysis of LC3 and p62 and ii) microscopic observation of GFP-LC3 puncta $(16,17)$.

Statistical methods for clinical correlation. Progression-free and overall survivals were calculated from the date of diagnosis to the date of first relapse or the last follow-up. Age and performance status distributions were similar between patients who did and did not express Beclin 1. The data were plotted as Kaplan-Meier curves, and the statistical significance was determined by the log-rank test. Data were censored when patients were lost to follow-up. Student's t-test (comparison of two groups) or one-way analysis of variance (ANOVA; comparison of more than two groups) were used to evaluate numerical data.

\section{Results}

CDDP induces cytoprotective autophagy in OCCC cell lines. To determine the effect of CDDP on autophagy and the role of autophagy in determining the sensitivity of cancer cells to the 

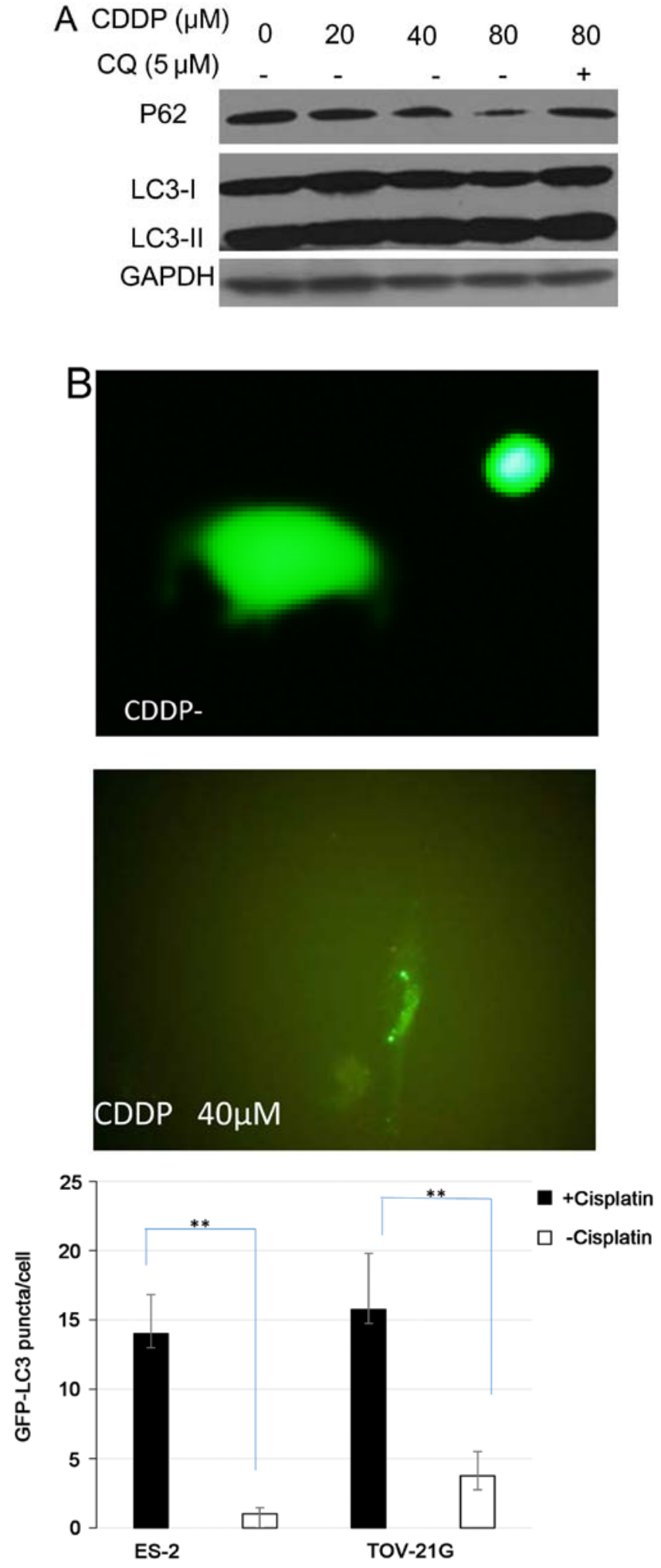

Figure 1. CDDP induces autophagy in OCCC cells. (A) ES-2 cells were treated with the indicated concentrations of cisplatin for $24 \mathrm{~h}$ in the absence or presence of $5 \mu \mathrm{M}$ of chloroquine. At the end of treatment, cell lysates were prepared, resolved by SDS-polyacrylamide gel electrophoresis and subjected to western blot analysis using anti-LC3, anti-p62 or anti-GAPDH antibodies, respectively. GAPDH was used as a loading control. (B) ES-2 and TOV-21G cells were transfected with a GFP-LC3 plasmid, followed by treatment with the indicated concentrations of CDDP for $24 \mathrm{~h}$. At the end of treatment, the cells were inspected under a fluorescence microscope. (C) Quantitation of the GFP-LC3 puncta was performed by counting 20 cells for each sample, and average numbers of puncta per cell are shown. The bars are the mean \pm SD of triplicate determinations; results shown are the representative of three identical experiments. ${ }^{* *} \mathrm{P}<0.01, \mathrm{t}$-test, CDDP vs. vehicle. drug, we first examined the activity of autophagy in OCCC cell lines. As shown in Fig. 1A, treatment of the human OCCC cell lines ES-2 and TOV-21G with CDDP produced a dosedependent activation of autophagy, as evidenced by increases in the amount of LC3 II and decreases in the amount of p62, two selective markers of autophagy. Fig. 1A also shows that LC3 II levels were further elevated in the presence of chloroquine, an inhibitor of autophagosome-lysosome fusion and LC3 II degradation, which is indicative of increased autophagic flux in the CDDP-treated cells. The stimulative effect of CDDP on autophagy was verified using a green fluorescent protein (GFP)-LC3 puncta-formation assay, which revealed an increase in the number of GFP-LC3 puncta in tumor cells treated with CDDP (Fig. 1B and C).

Inhibition of autophagy by chloroquine or Beclin 1 siRNA does not enhance sensitivity of OCCC cell lines to CDDP. To address the question of whether CDDP-stimulated autophagy has a protective or sensitizing role in CDDP-treated tumors, we compared cytotoxicity under a variety of drug treatment conditions. Concomitant treatment of OCCC cells with CDDP and inhibitors of autophagy, chloroquine did not enhance the cytotoxicity (Fig. 2A and B) induced by CDDP. The effect of autophagy on the CDDP-sensitivity of tumor cells was further explored by suppressing the expression of autophagy-related gene Beclin 1 and measuring the effect on tumor cell clonogenicity. Fig. 2C and D shows that knockdown of Beclin 1 in ES-2 and TOV-21G cells did not enhance CDDP-induced cytotoxicity. These results suggest that CDDP induces canonical autophagy. However, induction of CDDP-induced autophagy does not have a protective role in OCCC cell lines subjected to CDDP cytotoxicity.

Relationship between Beclin 1 protein expression and clinicopathological factors in OCCCs. Because Beclin 1 is a key regulator of autophagy, we focused on the relationship between Beclin 1 expression and clinicopathological factors in OCCCs. Loss of Beclin 1 expression (Beclin 1 immunointensity 0+) was observed in 38.3\% (23/60) of analyzed tumors (Fig. 3A and B). Patients were stratified into one of two groups depending on their status as determined by Beclin 1 immunostaining. The relationships between Beclin 1 protein expression and clinicopathological factors are shown in Table I. There were no significant correlations between loss of Beclin 1 expression and FIGO stage, CA125 levels, patient age, status of endometriosis, Ki-67 labeling index, chemotherapy regimen, or status of residual tumor. There was a marginally significant correlation between loss of Beclin 1 expression and lymph node metastasis $(\mathrm{P}=0.0579)$.

Relationship between loss of Beclin 1 expression and status of Kras and PIK3CA mutation, status of ZNF217 amplification, and status of ARIDIA expression in OCCCs. The 60 tumor samples in the present study had been characterized previously to determine mutational (Kras and PIK3CA) amplification (ZNF217) and immunohistochemical (ARID1A) status (11-13). Statistical analysis showed no correlations between loss of Beclin 1 expression and Kras and PIK3CA mutation status in OCCCs (Table II). In contrast, loss of Beclin 1 expression was significantly correlated with $Z N F 217$ amplification $(\mathrm{P}=0.024)$, 

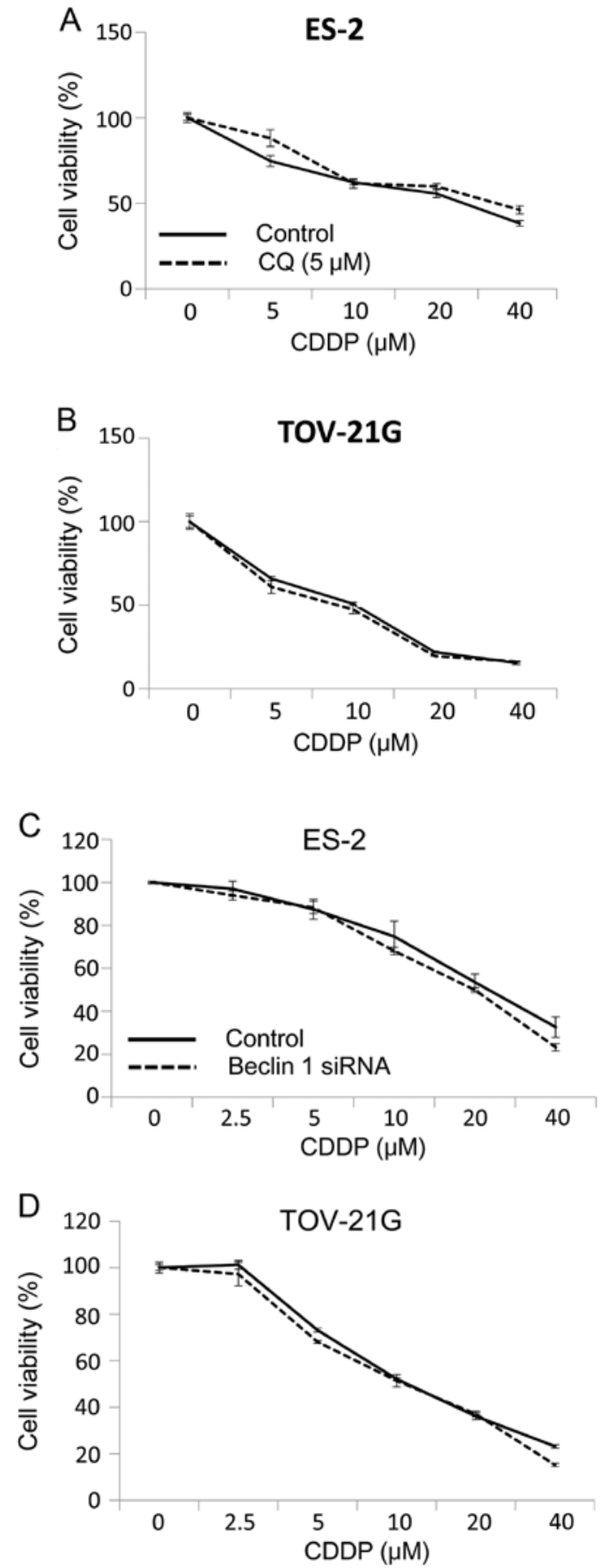

Figure 2. Inhibition of autophagy by chloroquine or Beclin 1 siRNA did not enhance sensitivity of OCCC cells to CDDP. (A-D) ES-2 and Tov-21G cells were treated with the indicated concentrations of cisplatin for $48 \mathrm{~h}$ in the presence or absence of chloroquine $(5 \mu \mathrm{M})$ or Beclin 1 siRNA. At the end of the treatment, cell viability was measured using an MTT assay.

and tended to correlate with loss of ARID1A expression in OCCCs $(\mathrm{P}=0.057)$ (Table II).

Effect of loss of Beclin 1 protein expression on progressionfree survival. We examined the effect of loss of Beclin 1 protein expression on the prognosis for progression-free survival. Kaplan-Meier estimates of progression-free/overall survival are plotted in Fig. 2. Of the 60 patients diagnosed at stages I-IV, 23 patients with loss of Beclin 1 expression had a shorter
Table I. Association between Beclin 1 expression and clinicopathological factors in patients with ovarian clear cell carcinoma.

\section{Beclin 1 \\ immunostaining}

Factors

Patients Negative Positive P-value

FIGO stage

$\begin{array}{lrrrr}\text { I, II } & 45 & 14 & 30 & 0.0852 \\ \text { III, IV } & 15 & 9 & 7 & \\ \begin{array}{l}\text { CA125 U/ml } \\ \quad<90\end{array} & & & & \\ \geq 90 & 30 & 9 & 21 & 0.1843 \\ \text { Age (years) } & 30 & 14 & 16 & \\ \quad<54 & & & & \\ \geq 54 & 30 & 12 & 18 & 0.7906 \\ & 30 & 11 & 19 & \end{array}$

Endometriosis

$\begin{array}{lrrrr}\text { Without } & 32 & 15 & 17 & 0.8848 \\ \text { With } & 28 & 8 & 20 & \\ \text { Ki-67 } & & & & \\ \text { Low } & 30 & 14 & 16 & 0.1843 \\ \text { High } & 30 & 9 & 21 & \\ \text { Residual tumor (cm) } & & & & \\ \quad<2 & 48 & 6 & 42 & 0.2781 \\ \geq 2 & 12 & 3 & 9 & \\ \text { Lymph node metastasis } & & & & \\ \quad \text { Negative } & 51 & 17 & 34 & 0.0579 \\ \text { Positive } & 9 & 6 & 3 & \\ \end{array}$

Table II. Association between Beclin 1 expression and status of ARID1A, K-ras, PIK3CA and ZNF217 in patients with ovarian clear cell carcinoma.

\begin{tabular}{lrrrr}
\hline & & \multicolumn{2}{c}{ Beclin 1 expression } & \\
\cline { 3 - 4 } Factors & Patients & Negative & Positive & P-value \\
\hline ARID1A & & & & \\
Negative & 9 & 6 & 3 & 0.0579 \\
Positive & 51 & 17 & 34 & \\
K-ras & & & & \\
Wild-type & 35 & 11 & 24 & 0.3443 \\
Mutant & 2 & 0 & 2 & \\
PIK3CA & & & & \\
Wild-type & 40 & 14 & 26 & 0.5412 \\
Mutant & 16 & 7 & 9 & \\
ZNF217 & & & & \\
Normal & 48 & 15 & 33 & 0.024 \\
Amplification & 12 & 8 & 4 & \\
\hline
\end{tabular}

progression-free survival than those with positive Beclin 1 expression ( $\mathrm{P}=0.027$; log-rank test) (Fig. 3C). Univariate 

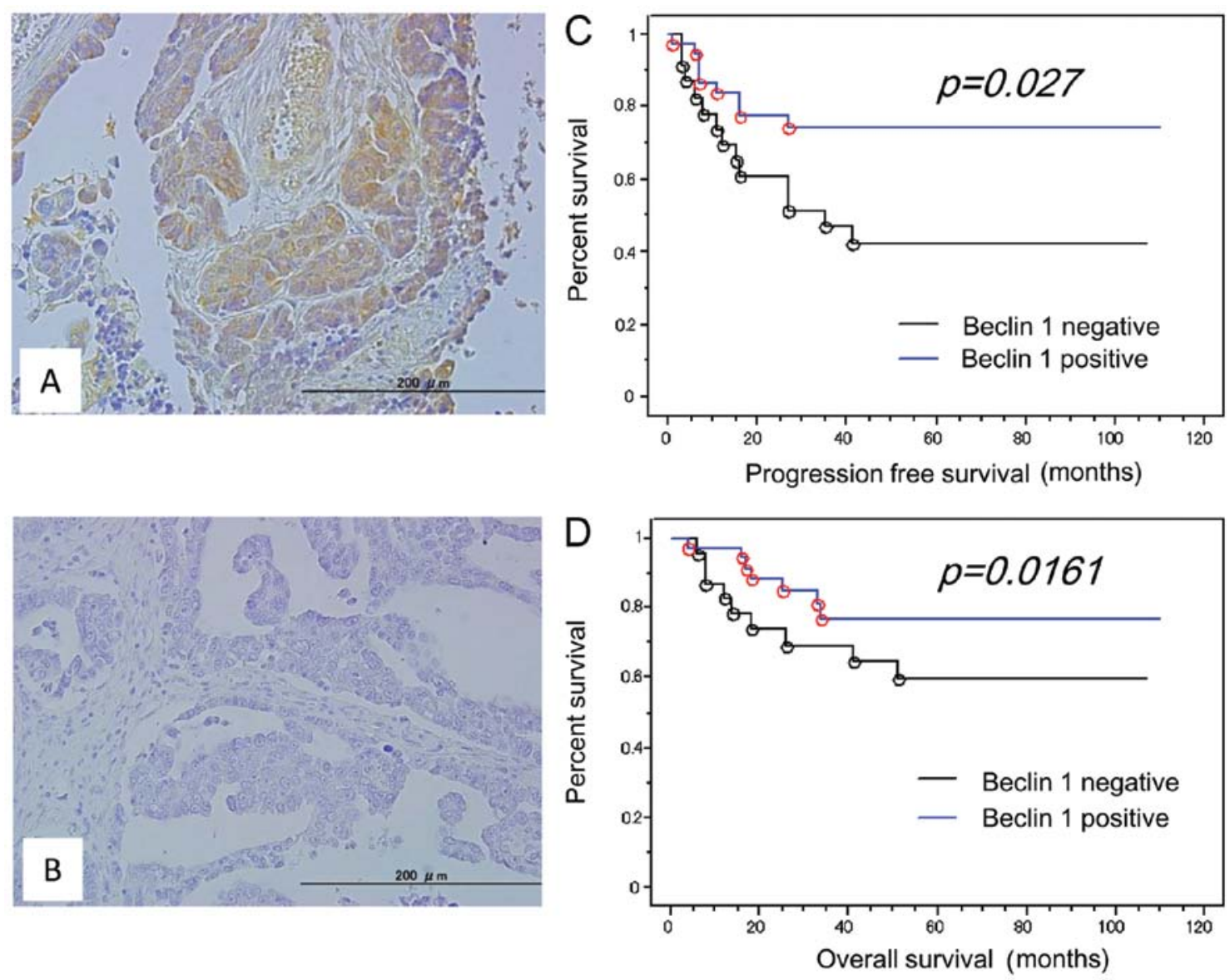

Figure 3. Immunoreactivity of Beclin 1 in OCCC tissues. (A) Intense Beclin 1 immunoreactivity in the cytoplasm of OCCC cells (Upper left panel). (B) An OCCC case with negative staining of Beclin 1. (C) Kaplan-Meier survival analysis showing that negative expression of Beclin 1 was associated with a shorter progression-free survival in comparison to positive Beclin 1 expression in OCCC who received cytoreductive surgery, followed by a standard platinum-based chemotherapy regimen ( $\mathrm{P}=0.027, \log -$ rank test). (D) Loss of Beclin 1 expression tended to correlate with shorter overall survival in patients with OCCC who received cytoreductive surgery, followed by a standard platinum-based chemotherapy regimen $(\mathrm{P}=0.161, \log$-rank test).

analysis demonstrated that FIGO stage III, IV $(\mathrm{P}<0.01$; log-rank test), CA125 levels ( $\mathrm{P}=0.01 ;$ log-rank test), residual tumor $(\geq 2 \mathrm{~cm})(\mathrm{P}<0.01$; log-rank test), and loss of Beclin 1 expression $(\mathrm{P}=0.027$; log-rank test) correlated with shorter progression-free survival. When the data were stratified for multivariate analysis, only residual tumor $(\geq 2 \mathrm{~cm})$ remained a significant $(\mathrm{P}=0.03)$ factor for shorter disease-free survival (data not shown).

Effect of loss of Beclin 1 protein expression on overall survival. Loss of Beclin 1 expression also tended to correlate with shorter overall survival in OCCC patients treated with platinum-based chemotherapy $(\mathrm{P}=0.161$; log-rank test $)$ (Fig. 3D). Univariate analysis demonstrated that FIGO stage III, IV ( $\mathrm{P}<0.01$; log-rank test), CA125 levels $(\mathrm{P}=0.01$; log-rank test), and residual tumor $(\geq 2 \mathrm{~cm})(\mathrm{P}<0.01$; log-rank test) significantly correlated with shorter overall survival. When these data were stratified for multivariate analysis, only residual tumor $(\geq 2 \mathrm{~cm})$ remained a significant $(\mathrm{P}=0.04)$ predictor for shorter overall survival (data not shown).

Relationship between Beclin 1 expression and chemotherapeutic response. Of the 60 OCCC patients, 14 had measurable residual disease following primary cytoreductive
Table III. The relationship between Becin 1 expression and platinum-based chemotherapeutic response.

\begin{tabular}{lccc}
\hline & Responder n (\%) & Non-responder n (\%) & P-value \\
\hline Negative & $4(50.0)$ & $4(50.0)$ & 0.937 \\
Positive & $2(33.3)$ & $4(66.7)$ & \\
\hline
\end{tabular}

surgery. Of these 14 patients, $6(42.8 \%)$ responded to chemotherapy and $8(57.2 \%)$ did not. Beclin 1-negative tumors were not more resistant to primary adjuvant chemotherapy than the Beclin 1-positive tumors (50.0 vs. $66.7 \%, \mathrm{P}=0.937)$ (Table III).

Beclin 1 knockdown increases growth, but not cell migration and invasion in OCCC cells. The findings of the present study suggest that Beclin 1 is a potential tumor suppressor in OCCCs. To assess the contribution of Beclin 1 expression to OCCC cell growth and survival, OCCC cell lines were treated with Beclin 1 siRNA and Beclin 1 levels and cell growth were assessed. Following Beclin 1 knockdown (Fig. 4A), cell growth increased in ES-2 and TOV-21G OCCC cell lines with positive Beclin 1 expression (Fig. 4B and C). However, there 

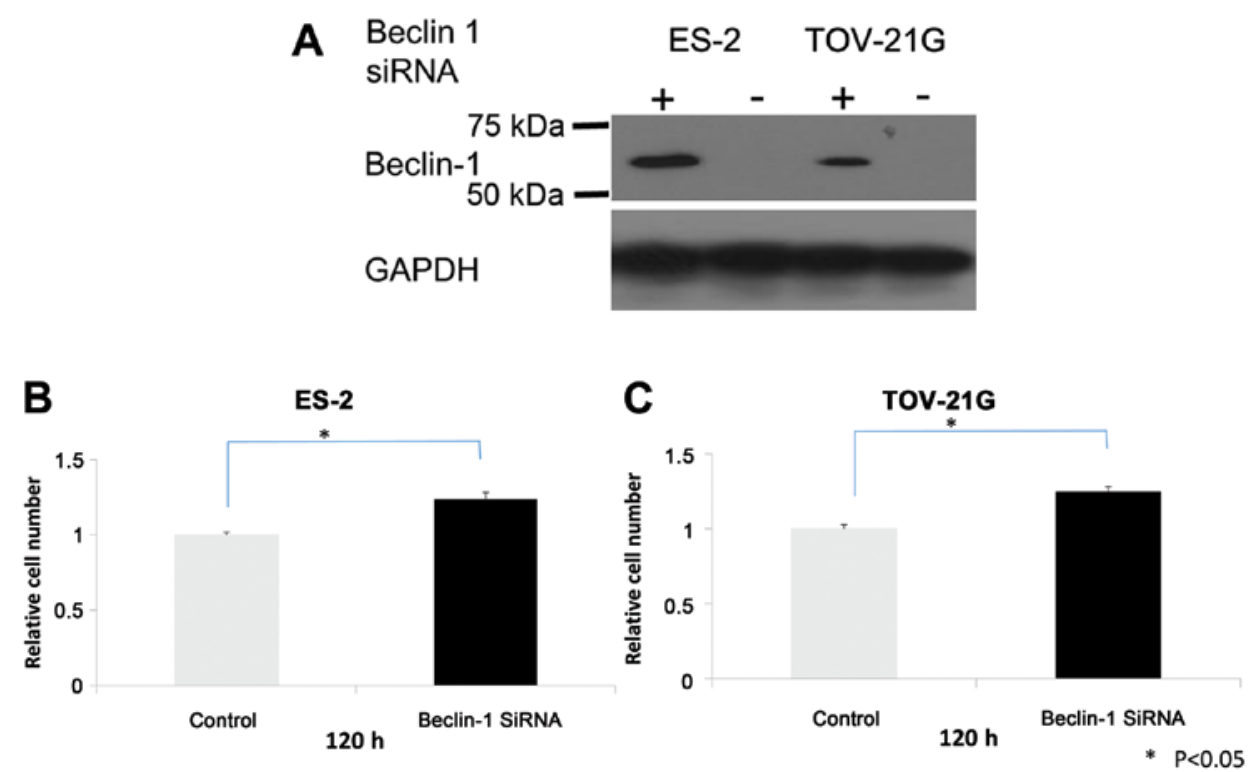

Figure 4. Effects of Beclin 1 knockdown on cell proliferation in OCCC cell lines. (A) Western blot analysis showing a significant reduction of Beclin 1 protein in Beclin 1 siRNA-treated cells compared with control siRNA-treated cells. (B and C) Effects of Beclin 1 gene knockdown. Beclin 1 knockdown significantly increased cell number in TOV-21G and ES-2 cells that harbor Beclin 1 expression.

was no profound inhibition or activation of cell migration and invasion observed in Beclin 1 siRNA-treated OCCC cells (data not shown).

\section{Discussion}

OCCCs, which comprise approximately $25 \%$ of all ovarian carcinomas in Japan, display a distinct gene expression profile relative to other histological types $(18,19)$. They are more aggressive and carry a worse prognosis than stage-matched serous adenocarcinomas $(20,21)$, possibly because OCCC is frequently refractory to platinum-based chemotherapy $(20,21)$. The mechanism of CDDP resistance in OCCC is still unclear. Therefore, in the present study, we first investigated whether autophagy is related to CDDP sensitivity in OCCC cell lines using autophagy inhibitors chloroquine or Beclin 1 siRNA. However, we did not find a relationship between autophagy and CDDP sensitivity, although several recent reports state that autophagy is related to chemoresistance in several types of cancer $(7,8)$. This discrepancy may be due to differences in organ-specific oncogenic pathways. Consequently, autophagy may not be an important factor in the OCCC carcinogenesis pathway.

We next focused on the relationship between Beclin 1 expression and clinicopathological, prognostic significance in OCCCs. Our most notable finding is that loss of Beclin 1 in OCCCs predicted a shorter progression-free interval. To date, there are only few molecular markers that predict the risk of early tumor recurrence in OCCCs $(22,23)$. Therefore, loss of Beclin 1 expression may be useful, alone or in combination with other markers, in identifying OCCC patients who are more susceptible to early recurrence. This is important because at least $60 \%$ of advanced-stage OCCC patients with a complete response to primary therapy ultimately develop recurrent disease (24). These observations could have an impact on clinical management. Patients with recurrent OCCCs derive the most benefit from secondary cytoreduction if recurrent tumors are small and localized (24-28). Therefore, patients who lack Beclin 1 expression could then be followed more frequently in order to detect recurrences early enough to benefit from either secondary cytoreductive surgery or second line chemotherapy. This indicates that the immunohistochemical analysis of Beclin 1 may be a useful predictor to find OCCC patients who tend to recur in the clinical setting.

In the present study, all patients were treated with either platinum and taxane combination or platinum and CPT-11 combination as the primary adjuvant regimen. Patients whose disease recurred after primary platinum-based chemotherapy were then treated with various second line chemotherapy agents, including liposomal doxorubicin, gemcitabine and docetaxel. Differing responses to these agents may have masked the effect of loss of Beclin 1 expression on overall survival in OCCC patients. In the present study, patients with Beclin 1-negative tumors did not have a significantly inferior response to chemotherapy when compared with patients who had Beclin 1-positive tumors. This clinical finding is consistent with current in vitro results showing that autophagy is not related to CDDP resistance. Furthermore, our in vitro Beclin 1 knockdown experiment also showed that loss of Beclin 1 expression was not related to OCCC metastasis. Therefore, the loss of Beclin 1 expression may be masking another mechanism related to shorter progression-free survival in OCCC.

Our in vitro data showed that silencing Beclin 1 using siRNA enhances cell growth in OCCC cell lines. Previous reports showed that autophagy also plays a role in tumorigenesis. For example, the essential autophagy regulator Beclin 1 is monoallelically deleted in human ovarian, breast and prostate cancers $(29,30)$. In addition, Beclin $1^{+/}$or Atg $4 \mathrm{C}^{-/}$mice are prone to tumors (31-33). Paradoxically, these findings suggest that the loss of a survival pathway enhances tumor growth. Recent studies have shown that simultaneous defects in 
autophagy and apoptosis activate the DNA damage response in vitro, promote gene amplification and aneuploidy, and accelerate mammary tumorigenesis $(34,35)$. Thus, loss of the prosurvival role of autophagy caused by a defect in Beclin 1 expression is likely to contribute to tumor progression by promoting genome damage and instability in OCCC development.

Molecular genetic evidence suggests that OCCCs develop as the result of a multistep process of oncogenic activation and tumor suppressor inactivation $(36,37)$. In this study, we focused on some of the important genetic events of OCCCs: inactivation of the tumor suppressor gene ARIDIA, gene amplification of the potential oncogene $Z N F 217$, and oncogenic mutation of Kras and PIK3CA (11-13). In addition, we also analyzed the association between these genetic events and loss of Beclin 1 expression. No significant relationship was observed between oncogenic mutation of Kras/PIK3CA and loss of Beclin 1 expression, which indicates that the loss of autophagy caused by loss of Beclin 1 expression is independent of these carcinogenesis events in OCCCs. Also, loss of Beclin 1 expression was significantly correlated with ZNF217 amplification and tended to be correlated with loss of ARIDIA expression. However, the significance of these relationships during OCCC development remains unclear. Thus, further studies are required to fully explore the relationship between the loss of Beclin 1 expression and these genetic events in vitro.

To the best of our knowledge, this is the first report suggesting that loss of Beclin 1 expression is a marker of poor progression-free survival in OCCCs. This study is limited by its small size given the relative rarity of OCCC. Larger prospective trials are needed to confirm our findings and to more fully explore the role of Beclin 1 in OCCC behavior.

In conclusion, the present study shows that autophagy defects caused by loss of Beclin 1 may be associated with malignant phenotype and poor prognosis of OCCC.

\section{Acknowledgements}

The present study was supported by grants from the Ministry of Education, Culture, Sports, Science and Technology in Japan.

\section{References}

1. Siegel R, Naishadham D and Jemal A: Cancer statistics for Hispanics/Latinos, 2012. CA Cancer J Clin 62: 283-298, 2012.

2. Cho KR and Shih IeM: Ovarian cancer. Annu Rev Pathol 4: 287-313, 2009.

3. Mandai M, Matsumura N, Baba T, Yamaguchi K, Hamanishi J and Konishi I: Ovarian clear cell carcinoma as a stress-responsive cancer: Influence of the microenvironment on the carcinogenesis and cancer phenotype. Cancer Lett 310: 129-133, 2011.

4. Hanahan D and Weinberg RA: The hallmarks of cancer. Cell 100: 57-70, 2000.

5. Shimizu S, Kanaseki T, Mizushima N, Mizuta T, ArakawaKobayashi S, Thompson CB and Tsujimoto Y: Role of Bcl-2 family proteins in a non-apoptotic programmed cell death dependent on autophagy genes. Nat Cell Biol 6: 1221-1228, 2004.

6. Rashmi R, Pillai SG, Vijayalingam S, Ryerse J and Chinnadurai G: $\mathrm{BH} 3$-only protein $\mathrm{BIK}$ induces caspase-independent cell death with autophagic features in Bcl-2 null cells. Oncogene 27: $1366-1375,2008$

7. Furuya D, Tsuji N, Yagihashi A and Watanabe N: Beclin 1 augmented cis-diamminedichloroplatinum induced apoptosis via enhancing caspase-9 activity. Exp Cell Res 307: 26-40, 2005.
8. Sun Y, Liu JH, Jin L, Pan L, Sui YX, Yang Y and Shi H: Beclin 1 influences cisplatin-induced apoptosis in cervical cancer CaSki cells by mitochondrial dependent pathway. Int J Gynecol Cancer 22: 1118-1124, 2012

9. Wang J: Beclin 1 bridges autophagy, apoptosis and differentiation. Autophagy 4: 947-948, 2008.

10. Eskelinen EL and Saftig P: Autophagy: A lysosomal degradation pathway with a central role in health and disease. Biochim Biophys Acta 1793: 664-673, 2009.

11. Katagiri A, Nakayama K, Rahman MT, Rahman M, Katagiri H, Nakayama N, Ishikawa M, Ishibashi T, Iida K, Kobayashi H, et al: Loss of ARID1A expression is related to shorter progression-free survival and chemoresistance in ovarian clear cell carcinoma. Mod Pathol 25: 282-288, 2012.

12. Rahman M, Nakayama K, Rahman MT, Nakayama N, Ishikawa M, Katagiri A, Iida K, Nakayama S, Otsuki Y, Shih IeM, et al: Clinicopathologic and biological analysis of PIK3CA mutation in ovarian clear cell carcinoma. Hum Pathol 43: 2197-2206, 2012.

13. Rahman MT, Nakayama K, Rahman M, Nakayama N, Ishikawa M, Katagiri A, Iida K, Nakayama S, Otsuki Y, Shih IeM, et al: Prognostic and therapeutic impact of the chromosome 20q13.2 ZNF217 locus amplification in ovarian clear cell carcinoma. Cancer 118: 2846-2857, 2012.

14. Nakayama K, Nakayama N, Jinawath N, Salani R, Kurman RJ, Shih IeM and Wang TL: Amplicon profiles in ovarian serous carcinomas. Int J Cancer 120: 2613-2617, 2007.

15. Nakayama K, Miyazaki K, Kanzaki A, Fukumoto $M$ and Takebayashi Y: Expression and cisplatin sensitivity of coppertransporting P-type adenosine triphosphatase (ATP7B) in human solid carcinoma cell lines. Oncol Rep 8: 1285-1287, 2001.

16. Wu H, Zhu H, Liu DX, Niu TK, Ren X, Patel R, Hait WN and Yang JM: Silencing of elongation factor-2 kinase potentiates the effect of 2-deoxy-D-glucose against human glioma cells through blunting of autophagy. Cancer Res 69: 2453-2460, 2009.

17. Cheng Y, Li H, Ren X, Niu T, Hait WN and Yang J: Cytoprotective effect of the elongation factor-2 kinase-mediated autophagy in breast cancer cells subjected to growth factor inhibition. PLoS One 5: e9715, 2010.

18. Schwartz DR, Kardia SL, Shedden KA, Kuick R, Michailidis G, Taylor JM, Misek DE, Wu R, Zhai Y, Darrah DM, et al: Gene expression in ovarian cancer reflects both morphology and biological behavior, distinguishing clear cell from other poorprognosis ovarian carcinomas. Cancer Res 62: 4722-4729, 2002.

19. Yamaguchi K, Mandai M, Oura T, Matsumura N, Hamanishi J, Baba T, Matsui S, Murphy SK and Konishi I: Identification of an ovarian clear cell carcinoma gene signature that reflects inherent disease biology and the carcinogenic processes. Oncogene 29: 1741-1752, 2010.

20. Sugiyama T, Kamura T, Kigawa J, Terakawa N, Kikuchi Y, Kita T, Suzuki M, Sato I and Taguchi K: Clinical characteristics of clear cell carcinoma of the ovary: A distinct histologic type with poor prognosis and resistance to platinum-based chemotherapy. Cancer 88: 2584-2589, 2000.

21. Goff BA, Sainz de la Cuesta R, Muntz HG, Fleischhacker D, Ek M, Rice LW, Nikrui N, Tamimi HK, Cain JM, Greer BE, et al: Clear cell carcinoma of the ovary: A distinct histologic type with poor prognosis and resistance to platinum-based chemotherapy in stage III disease. Gynecol Oncol 60: 412-417, 1996.

22. Köbel M, Xu H, Bourne PA, Spaulding BO, Shih IeM, Mao TL, Soslow RA, Ewanowich CA, Kalloger SE, Mehl E, et al: IGF2BP3 (IMP3) expression is a marker of unfavorable prognosis in ovarian carcinoma of clear cell subtype. Mod Pathol 22: 469-475, 2009.

23. Ho CM, Cheng WF, Lin MC, Chen TC, Huang SH, Liu FS, Chien CC, Yu MH, Wang TY and Hsieh CY: Prognostic and predictive values of E-cadherin for patients of ovarian clear cell adenocarcinoma. Int J Gynecol Cancer 20: 1490-1497, 2010.

24. Díaz-Montes TP and Bristow RE: Secondary cytoreduction for patients with recurrent ovarian cancer. Curr Oncol Rep 7: 451-458, 2005.

25. Harter $\mathrm{P}$ and du Bois A: The role of surgery in ovarian cancer with special emphasis on cytoreductive surgery for recurrence. Curr Opin Oncol 17: 505-514, 2005.

26. Gadducci A, Iacconi P, Cosio S, Fanucchi A, Cristofani R and Genazzani AR: Complete salvage surgical cytoreduction improves further survival of patients with late recurrent ovarian cancer. Gynecol Oncol 79: 344-349, 2000. 
27. Gadducci A, Iacconi P, Fanucchi A, Cosio S, Teti G and Genazzani AR: Surgical cytoreduction during second-look laparotomy in patients with advanced ovarian cancer. Anticancer Res 20: 1959-1964, 2000.

28. Zang RY, Li ZT, Tang J, Cheng X, Cai SM, Zhang ZY and Teng NN: Secondary cytoreductive surgery for patients with relapsed epithelial ovarian carcinoma: Who benefits? Cancer 100: 1152-1161, 2004

29. Liang XH, Jackson S, Seaman M, Brown K, Kempkes B, Hibshoosh $\mathrm{H}$ and Levine B: Induction of autophagy and inhibition of tumorigenesis by beclin 1. Nature 402: 672-676, 1999.

30. Aita VM, Liang XH, Murty VV, Pincus DL, Yu W, Cayanis E, Kalachikov S, Gilliam TC and Levine B: Cloning and genomic organization of beclin 1, a candidate tumor suppressor gene on chromosome 17q21. Genomics 59: 59-65, 1999.

31. Qu X, Yu J, Bhagat G, Furuya N, Hibshoosh H, Troxel A, Rosen J, Eskelinen EL, Mizushima N, Ohsumi Y, et al: Promotion of tumorigenesis by heterozygous disruption of the beclin 1 autophagy gene. J Clin Invest 112: 1809-1820, 2003.

32. Yue Z, Jin S, Yang C, Levine AJ and Heintz N: Beclin 1, an autophagy gene essential for early embryonic development, is a haploinsufficient tumor suppressor. Proc Natl Acad Sci USA 100: $15077-15082,2003$
33. Mariño G, Salvador-Montoliu N, Fueyo A, Knecht E, Mizushima N and López-Otín C: Tissue-specific autophagy alterations and increased tumorigenesis in mice deficient in Atg4C/autophagin-3. J Biol Chem 282: 18573-18583, 2007.

34. Karantza-Wadsworth V, Patel S, Kravchuk O, Chen G, Mathew R, Jin S and White E: Autophagy mitigates metabolic stress and genome damage in mammary tumorigenesis. Genes Dev 21: 1621-1635, 2007

35. Mathew R, Kongara S, Beaudoin B, Karp CM, Bray K, Degenhardt K, Chen G, Jin S and White E: Autophagy suppresses tumor progression by limiting chromosomal instability. Genes Dev 21: 1367-1381, 2007.

36. Kuo KT, Mao TL, Jones S, Veras E, Ayhan A, Wang TL, Glas R, Slamon D, Velculescu VE, Kuman RJ, et al: Frequent activating mutations of PIK3CA in ovarian clear cell carcinoma. Am J Pathol 174: 1597-1601, 2009.

37. Jones S, Wang TL, Shih IeM, Mao TL, Nakayama K, Roden R, Glas R, Slamon D, Diaz LA Jr, Vogelstein B, et al: Frequent mutations of chromatin remodeling gene ARID1A in ovarian clear cell carcinoma. Science 330: 228-231, 2010. 